\title{
Evaluation of Dark Current Profile for Prediction of Voltage Holding Capability on Multi-Aperture Multi-Grid Accelerator for ITER $^{*}$
}

\author{
Ryo NISHIKIORI, Atsushi KOJIMA, Masaya HANADA, Mieko KASHIWAGI, \\ Kazuhiro WATANABE, Naotaka UMEDA, Hiroyuki TOBARI, Masafumi YOSHIDA, \\ Masahiro ICHIKAWA, Junichi HIRATSUKA, Yasushi YAMANO ${ }^{1)}$, Tetsuya OKURA ${ }^{1)}$ \\ and NB Heating Technology Group \\ Japan Atomic Energy Agency, 801-1 Mukoyama, Naka 311-0193, Japan \\ ${ }^{1)}$ Saitama University, 255 Shimo-Okubo, Sakura-ku, Saitama 338-8570, Japan
}

(Received 30 November 2015 / Accepted 18 January 2016)

\begin{abstract}
Dark current analysis to estimate the current density of the field-emitted electrons have been developed for the prediction of the yoltage holding capability of multi-aperture multi-grid accelerators used in JT-60SA and ITER neutral beam injectors. From the experimental measurement of the field-emitted electrons in multi-aperture multi-grid accelerators, $\beta E_{\mathrm{BD}}$, which represent the critical current density to trigger the breakdown, were found to be constant with around $6 \times 10^{3} \mathrm{kV} / \mathrm{mm}$ regardless of the electric field profiles and the surface conditions in an accelerator with a surface area of $0.1 \mathrm{~m}^{2}$. In addition, the dark current was measured in the region with the electric field above $\sim 60 \%$ of the electric field at the breakdown, which leads to the determination of the emission region in the analytical estimation of the dark current. Furthermore, from the measurement of $\beta E_{\mathrm{BD}}$ on electrodes with various surface area, $\beta E_{\mathrm{BD}}$ was found to decreases with an increase of the surface area $S\left(\beta E_{\mathrm{BD}}=4 \times\right.$ $10^{3} S^{-0.3}$ ). From these results, the dark current profile at the critical current density can be estimated from the electric field analysis, which leads to the development of the breakdown model and the prediction of the voltage holding capability on the multi-aperture multi-grid accelerators.
\end{abstract}

(C) 2016 The Japan Society of Plasma Science and Nuclear Fusion Research

Keywords: ITER, MAMuG, neutral beam injector, voltage holding capability, breakdown, field-emitted electron, field enhancement factor, effective electric field, dark current

DOI: $10.1585 / \mathrm{pfr} .11 .2401014$

\section{Introduction}

A neutral beam injector (NBI) with negative ion source and high-energy high-current electrostatic accelerator with multi-aperture multi-grid (MAMuG) concept [1] is indispensable for fusion plasma heating and current drive in future fusion reactors. For JT-60SA and ITER NBI systems, 22 - 40 A negative ion beams are required to be accelerated up to $0.5-1 \mathrm{MeV}[2,3]$. To achieve such highenergy high-current beams, one of the critical issues is the high voltage holding which is dominated by breakdown in accelerators.

Previously, the voltage holding capability of the MAMuG accelerators has been empirically investigated in terms of the geometric characteristics such as the area, the multi-aperture and the multi-grid effects $[4,5]$ because there is no reliable physics-based-model for the prediction of the voltage holding capability. In the past results, it was suggested that breakdown was triggered by dark current and the voltage holding capability followed Clump-

author'se-mail: nishikiori.ryo@jaea.go.jp

*) This article is based on the presentation at the 25 th International Toki Conference (ITC25). theory [6].

Therefore, an estimation of the dark current due to field-emitted electrons [7] is one of key techniques for the prediction of the voltage holding capability. However, there is no available technique to estimate the dark current profile in accelerators having large-area grid and a lot of aperture with locally-strong electric field.

This time, toward the development of the dark current analysis, the behavior of the dark current depending on the electric field and surface area was investigated by using accelerators having various electric field profiles.

\section{Evaluating Field-emitted Electrons}

In a vacuum condition, electrons are emitted from metal surface by applying high voltage according to Fowler-Nordheim (F-N) theory $[8,9]$. In this F-N theory, current density $j$ of field-emitted electrons are given by,

$$
\begin{aligned}
j= & \frac{1.54 \times 10^{-6}(\beta E)^{2}}{\phi t^{2}(y)} \\
& \times \exp \left\{\frac{-6.83 \times 10^{9} \phi^{3 / 2} v(y)}{\beta E}\right\},
\end{aligned}
$$


where $\beta$ is the field enhancement factor, $E$ is the electric field, $\phi$ is the work function, and $t(y)$ and $v(y)$ are the correction factors. Here, $E$ can be expressed as average electric field $E_{\text {ave }}$ in actual electrodes where $E_{\text {ave }}$ is the quotient of applied voltage $V$ and gap length $d$.

Eq. (1) is deformed as follows to evolve $\beta$ from experimentally-measured values:

$$
\begin{aligned}
\ln \left(\frac{I}{V^{2}}\right)= & -\frac{2.84 \times 10^{9} \phi^{3 / 2} d}{\beta} \frac{1}{V} \\
& +\ln \left\{1.54 \times 10^{-6} \frac{\beta^{2} A}{\phi d^{2}} \exp \left(\frac{10.4}{\phi^{1 / 2}}\right)\right\},
\end{aligned}
$$

where $A$ is the emission area in F-N theory. If the dark current is originated in field-emitted electrons, then the plot of $1 / V$ vs $\ln \left(I / V^{2}\right)$ become linear. As noticed from eq. (2), this plot called F-N plot gives $\beta$ with the gradient $M$ by,

$$
\beta=-\frac{2.84 \times 10^{9} \phi^{3 / 2} d}{M} .
$$

Therefore $\beta E_{\text {ave }}$, so-called effective electric field which is a key parameter to estimate the current density of the field-emitted electrons, has been determined from experiments. If $\beta E_{\text {ave }}$ can be evaluated without experiment, the dark current density can be analyzed.

By using miniaturized parallel electrodes, whose surface area is $1 / 6000$ of that for JT-60SA, $\beta E_{\text {ave }}$ is reported to be constant regardless of breakdown voltage [10]. However, the field distribution is complicated in actual MA$\mathrm{MuG}$ accelerator. Electric field is higher around the apertures in the grid with small surface area, and lower in the region of support where gap and surface area is larger than those of the grid as shown in Fig. 1. Therefore, it is necessary to investigate the influence of field distribution on $\beta E_{\text {ave. }}$.

In addition, in order to understand the dark current profile, lower limit of electric field for the field emission is also important. In the experiments, dark current can be measured only in the region with relatively strong electric field. This indicates that there is a certain limit of the electric field for the field emission and breakdown does not occur in the region where electric field is below the limit. By applying this lower limit, dark current analysis based on $\beta E_{\text {ave }}$ can be realized as shown in Fig. 1 where electrons are emitted from anywhere.

Toward the analytical estimation of the field-emitted electrons, these two issues were investigated.

\section{Experimental Setup}

Dedicated experiment for high voltage holding was carried out by using an acceleration of a 5-stage MAMuG accelerator for cathode and anode electrodes. Both electrodes are composed of copper grid, aluminum flange, and stainless steel support. Same grids with surface dimension of $204 \mathrm{~mm} \times 234 \mathrm{~mm}$ and 49 apertures were used in both electrodes. Diameter of flange and support was $796 \mathrm{~mm}$ for cathode and $430 \mathrm{~mm}$ for anode.

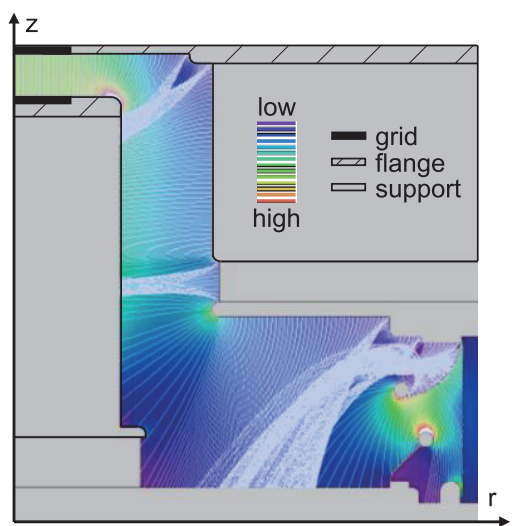

Fig. 1 Calculation result of electric field analysis in the $\mathrm{MeV}$ accelerator. Filled contour shows the electric field strength, and the line shows trajectory of electrons emitted from cathode surface. (a)

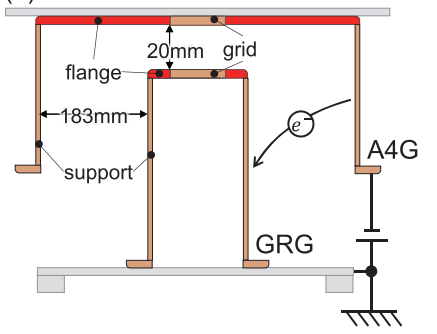

(b)

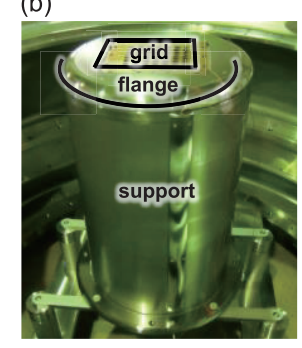

Fig. 2 (a) Schematic of experimental setup. High voltage was applied to cathode. (b) Photo of anode which is divided into 3 parts insulating from each other.

In this experiment, the anode was divided into 3 regions by insulating from each other to measure the dark current separately as shown in Fig. 2. These regions have different electric field profile and average electric field $E_{\text {ave }}$. Although $E_{\text {ave }}$ were same in grid and flange regions with gap length of $20 \mathrm{~mm}$, grid has about 3 times higher electric field around the apertures. Gap length in support region is $183 \mathrm{~mm}$, thus $E_{\text {ave }}$ is lower compared to grid and flange regions.

Negatively charged high voltage is applied to the cathode by a power supply with capability of $-300 \mathrm{kV}$ and $10 \mathrm{~mA}$ in a vacuum pressure below $4 \times 10^{-3} \mathrm{~Pa}$. Applied voltage was increased by $1 \mathrm{kV} / 1 \mathrm{sec}$ until breakdown occurs in a shot.

This shot is repeated until $V_{\mathrm{BD}}$ is saturated after the spark conditioning is fully satisfied. Dark currents in each region and applied voltage were measured in each shot and parameters of $V_{\mathrm{BD}}, V_{\mathrm{FE}}, I_{\mathrm{BD}}$, and $\beta$ were estimated as shown in Fig. 3. Here, $V_{\mathrm{BD}}$ is the voltage where breakdown occurs. $V_{\mathrm{FE}}$ is the lower limit of the voltage where dark current is firstly measured. $I_{\mathrm{BD}}$ is the dark current just before the breakdown occurs. $\beta$ is the field enhancement factor which is derived from eq. (3) with the gradient of F$\mathrm{N}$ plot as shown in Fig. 3 (b) and assuming work function 

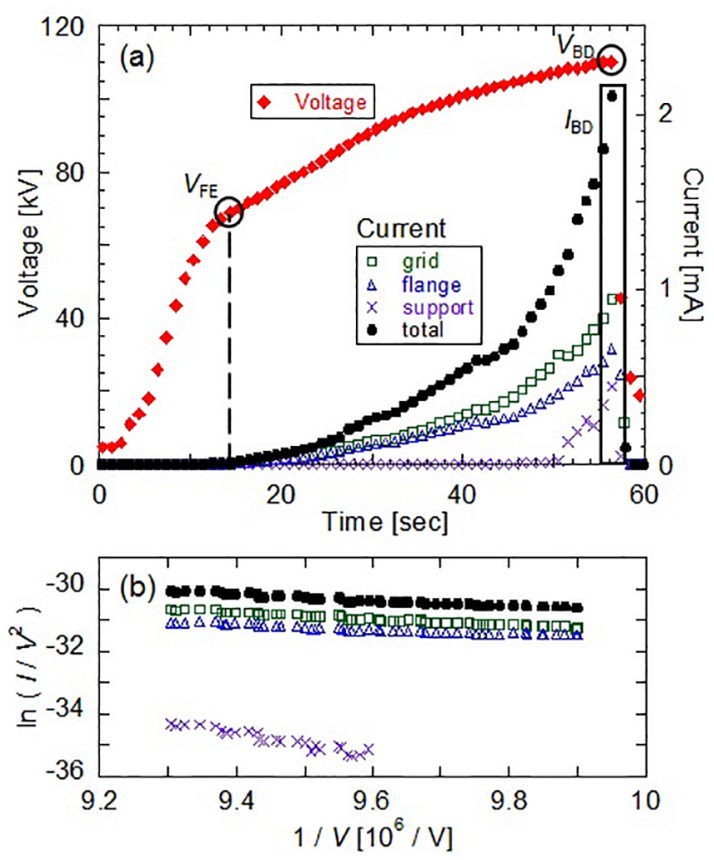

Fig. 3 (a) Typical voltage and current acquired from the experiment. (b) F-N plots made from acquired data for each region. Range of the plots depend on the range of the applied voltage where dark currents were observed.

of $4.6 \mathrm{eV}$ for copper [11], $4.3 \mathrm{eV}$ for aluminum [12], and for stainless steel [13] respectively.

\section{Experimental Result}

Figure 4 shows the history of the measured parameters in each shot.

$V_{\mathrm{BD}}$ increases up to $\sim 120 \mathrm{kV}$ in maximum as the spark conditioning progresses and $V_{\mathrm{FE}}$ varies along with $V_{\mathrm{BD}}$. Note that low $V_{\mathrm{FE}}$ due to the unstable condition after the breakdown is excluded.

$I_{\mathrm{BD}}$ for grid and flange were constant throughout the experiment with around $1 \mathrm{~mA}$. It seems that breakdown occurs in some probability when dark current is beyond the critical value which is independent of applied voltage. $I_{\mathrm{BD}}$ for support varies by the shot which have little involvement in breakdowns.

$\beta$ decreases in opposition to the increase of $V_{\mathrm{BD}}$ which is consistent with previous result [10]. It is thought to be that $\beta$ indicates the degree of progress of spark conditioning.

\subsection{Influence of electric field on $\boldsymbol{\beta} \boldsymbol{E}_{\text {ave }}$}

Figure 5 (a) shows $\beta E_{\text {ave }}$ in each region. When $E_{\text {ave }}^{\text {geid }}=$ $E_{\text {ave }}^{\text {fiange }}=E_{\mathrm{BD}}$ was defined because all the breakdowns occurred in either the grid or the flange which two has same gap lengths, then $\beta E_{\mathrm{BD}}$ were found to be same in both region with around $6 \times 10^{3} \mathrm{kV} / \mathrm{mm}$ which indicates that influence of the electric field around the apertures of the grid is included in $\beta$. Measured $\beta E_{\text {ave }}^{\text {support }}$ was smaller than the others with around $9 \times 10^{2} \mathrm{kV} / \mathrm{mm}$ because $\beta E_{\text {ave }}^{\text {support }}$ was

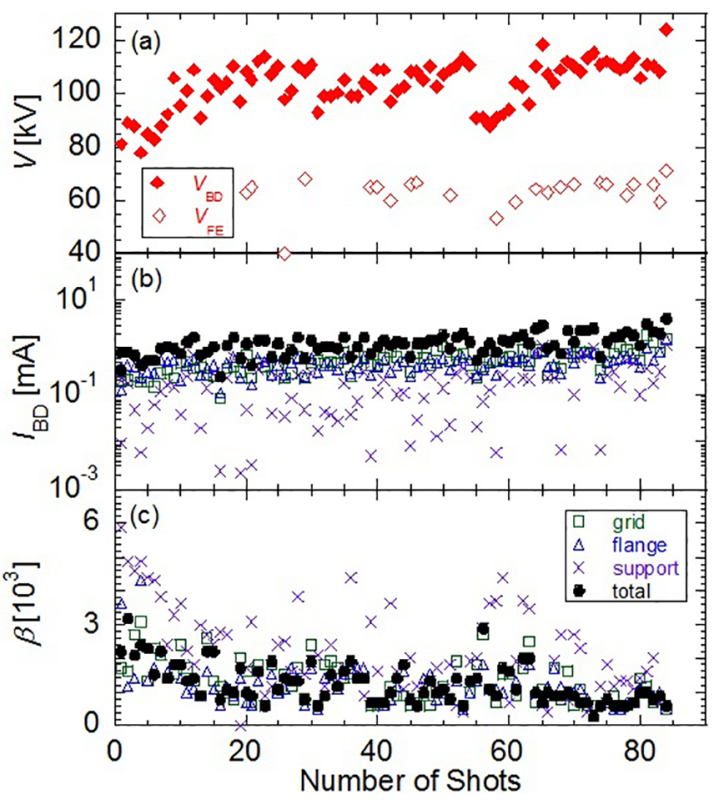

Fig. 4 Experimental result of (a) $V_{\mathrm{BD}}$ and $V_{\mathrm{FE}}$, (b) $I_{\mathrm{BD}}$ in each region, and (c) $\beta$ in each region in each shot.

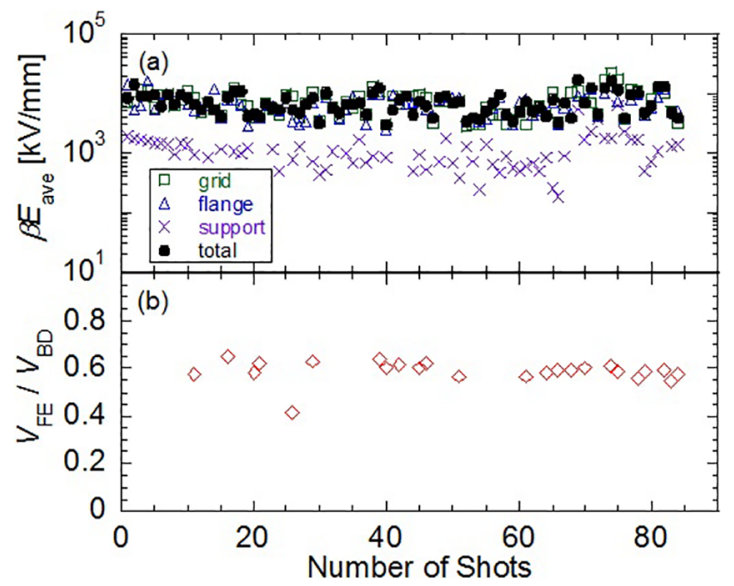

Fig. 5 Experimental result of (a) $\beta E_{\text {ave }}$ in each region and (b) $V_{\mathrm{FE}} / V_{\mathrm{BD}}$.

smaller than $E_{\mathrm{BD}}$, namely no breakdown was observed in the support region.

In addition, $\beta E_{\mathrm{BD}}$ were kept constant throughout the experiment which means that $\beta E_{\mathrm{BD}}$ is independent of the $V_{\mathrm{BD}}$. This result can be explained if the breakdown occurs in some probability when current density of the fieldemitted electrons is beyond the critical value which is independent of $V_{\mathrm{BD}}$.

\subsection{Lower limit of the field emission}

From the measurement of $V_{\mathrm{FE}}$, lower limit of the applied voltage for the field emission was found to be $\sim 60 \%$ of the $V_{\mathrm{BD}}$ regardless of $V_{\mathrm{BD}}$ as shown in Fig. 5 (b). This suggests that lower limit of the field emission depends on the condition of the accelerator and field-emitted electrons 


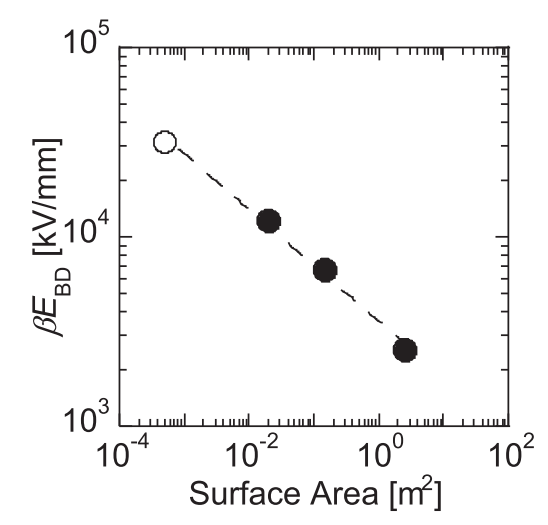

Fig. 6 Relation between surface area and measured $\beta E_{\mathrm{BD}}$ in various electrodes. Open circle is the result from miniaturized parallel electrodes [10].

which triggers the breakdown are produced in the region where surface electric field is higher than $0.6 E_{\mathrm{BD}}$. This is consistent with no breakdown observed in the support region where $E_{\text {ave }}$ is less than $0.6 E_{\mathrm{BD}}$.

\subsection{Surface area effect of current density}

From two results above, it can be concluded that $\beta E_{\mathrm{BD}}$, which is constant regardless of the breakdown voltage, shows the critical dark current density to trigger the breakdown and the region of $>0.6 E_{\mathrm{BD}}$ is related to be breakdown induced by the dark current. Therefore, critical current density of the field-emitted electrons to trigger the breakdown are expected to be estimated in the electric field analysis by using $\beta E_{\mathrm{BD}}$ within the region of $>0.6 E_{\mathrm{BD}}$.

By applying this assumption, surface area having influence on breakdown can be determined. Figure 6 shows the relation between surface area of various electrodes and measured $\beta E_{\mathrm{BD}}$ in each electrode. $\beta E_{\mathrm{BD}}$ decreases as surface area $S$ increases with $\beta E_{\mathrm{BD}}=4 \times 10^{3} S^{-0.3}$, which indicate that the critical dark current density for breakdown is reduced by increasing the surface area. This result agrees with the reduction of the voltage holding capability in empirical surface area effect. Therefore it is expected that $\beta E_{\mathrm{BD}}$ can be extrapolated from surface area leading to the estimation of field-emitted electrons in various electric field profile.

\section{Summary}

Analytical technique to estimate the current density of the field-emitted electrons have been developed for multiaperture multi-grid accelerators such as JT-60SA and ITER NBI. This time, dark currents were measured in variation of the electric fields to investigate $\beta E_{\text {ave }}$ and emission region of the electrons in actual MAMuG accelerators.

As the results, $\beta E_{\mathrm{BD}}$ that represents the critical dark current density were found to be around $6 \times 10^{3} \mathrm{kV} / \mathrm{mm}$ regardless of the regions and the conditions in an accelerator. Furthermore, $\beta E_{\mathrm{BD}}$ have dependence only on the surface area of the emission region with $\beta E_{\mathrm{BD}}=4 \times 10^{3} S^{-0.3}$ where electric field is above $60 \%$ of $E_{\mathrm{BD}}$.

The developed analytical estimation of dark current will lead to the development of the model of the breakdown process in accelerators which is necessary for the prediction of the voltage holding capability.

[1] M. Kashiwagi et al., Nucl. Fusion 49, 065008 (2009).

[2] R. Hemsworth et al., Nucl. Fusion 49, 045006 (2009).

[3] A. Kojima et al., Nucl. Fusion 51, 083049 (2011).

[4] A. Kojima et al., Rev. Sci. Instrum. 83, 02B117 (2012).

[5] A. Kojima et al., Rev. Sci. Instrum. 87, $02 B 304$ (2016).

[6] K. Watanabe et al., J. Appl. Phys. 72, 3949 (1992).

[7] A. Kojima et al., J. Vac. Soc. Jpn. 56, 502 (2013).

[8] R.H. Fowler and L.W. Nordheim, Proc. R. Soc. London A 119, 173 (1928).

[9] R.V. Latham, High-Voltage Vacuum Insulation: Basic Concepts and Technological Practice (Academic Press, London, 1995) pp.115-164.

[10] R. Ishida et al., IEEJ Trans. FM 134, 12, 622 (2014).

[11] E.W.J. Mitchell and J.W. Mitchell, Proc. R. Soc. London A 210, 70 (1951).

[12] R.M. Eastment and C.H.B. Mee, J. Phys. F 3, 1738 (1973).

[13] R.G. Wilson, J. Appl. Phys. 37, 2261 (1966). 